\title{
Carotenoproteins from the Exoskeleton and the Muscular Epithelium of the Black Tiger Prawn Penaeus monodon
}

\author{
Shah Amran Nur-E-Borhan, Shigeru Okada, Shugo Watabe, and Katsumi Yamaguchi \\ Laboratory of Marine Biochemistry, Faculty of Agriculture, The University of Tokyo, Yayoi, Bunkyo, Tokyo 113, \\ Japan
}

(Received October 3, 1994)

\begin{abstract}
A blue carotenoprotein $\left(\lambda_{\max }=615 \mathrm{~nm}\right)$ was purified and characterized from the exoskeleton of black tiger prawn Penaeus monodon. It contained free astaxanthin as the prosthetic group. Its molecular weight was found to be $126 \mathrm{kDa}$ and $120 \mathrm{kDa}$ by gel filtration and native-polyacrylamide gel electrophoresis (PAGE), respectively. SDS-PAGE indicated that the carotenoprotein consisted of six subunits, with three sets of two kinds of subunits of 22.4 and $21.4 \mathrm{kDa}$ in a molar ratio of $1: 1$. The stoichiometry of carotenoid and apoprotein was $2: 1$. Another blue carotenoprotein $\left(\lambda_{\max }=625 \mathrm{~nm}\right)$ was isolated from the muscular epithelium of $P$. monodon by collagenase treatment. The molecular weight of the carotenoprotein was determined to be $21.4 \mathrm{kDa}$ by gel filtration. SDS-PAGE indicated that the carotenoprotein was composed of a polypeptide of $22.9 \mathrm{kDa}$. This carotenoprotein also contained free astaxanthin as the prosthetic group.
\end{abstract}

Key words: black tiger prawn, carotenoprotein, astaxanthin, exoskeleton, muscular epithelium, collagenase

Carotenoproteins are widely distributed in invertebrates, especially in crustaceans and are implicated in their coloration. Many studies have been done on carotenoproteins, such as their distribution, ${ }^{1-3)}$ spectral shift of the prosthetic groups, ${ }^{4,5)}$ and molecular properties of the protein moiety. ${ }^{6,7)}$ Some carotenoproteins shift their absorption maxima to longer wavelengths by about $160 \mathrm{~nm}$ without changing the whole absorption pattern when the prosthetic groups are bound to the apoprotein. ${ }^{8,9)}$ This phenomenon is comparable to the alteration in absorption spectrum of retinal with and without its apoproteins. ${ }^{10)}$ The blue carotenoprotein, $\alpha$-crustacyanin, from the carapace of the lobster Homarus gammarus is one of the best models for such interaction between carotenoid and its associated protein. ${ }^{11-17}$

A few studies have been reported on carotenoproteins of commercially valuable aquatic animals. Muriana et al. reported a blue carotenoprotein $\left(\lambda_{\max }=640 \mathrm{~nm}\right)$ of 280 kDa from Penaeus japonicus. ${ }^{18)}$ Since the body color of prawn affects the market price, the color development largely due to the amount of carotenoid in the diet is the major concern of those involved in prawn aquaculture.

The black tiger prawn Penaeus monodon is also an important species in aquaculture. The appearance of this specimen is characterized as generally blue/black banded and sometimes slightly pale in color. Our previous work has indicated that the main carotenoid in the exoskeleton of $P$. momodon is astaxanthin even if the prawns show a variety of body colors. ${ }^{19)}$ The color turns red when the prawn is heated or treated with organic solvent. This color change suggests the presence of carotenoid as carotenoprotein in the exoskeleton and the muscular epithelium. Therefore carotenoprotein must have an important role for expression of the body color of $P$. monodon. This encouraged us to isolate and characterize carotenoproteins in the exoskeleton and the muscular epithelium of $P$. monodon.

\section{Materials and Methods}

\section{Materials}

Frozen specimens of black tiger prawn $P$. monodon were purchased at the Tokyo Central Wholesale Market, and stored at $-80^{\circ} \mathrm{C}$ until used.

\section{Purification of Carotenoprotein from the Exoskeleton}

The exoskeleton of the abdominal part was peeled from the prawn and the adhering hypodermic tissues were cleanly removed. The exoskeleton (about $110 \mathrm{~g}$ ) was then ground in an electric blender and washed for $4-5 \mathrm{~h}$ under continuous stirring with 10 volumes of $0.3 \mathrm{M}$ boric acid adjusted to pH 6.8 with solid Tris. The suspension was then filtered through No. 2 filter paper (Advantec Toyo) and the residue was subjected to the overnight extraction of carotenoproteins with 10 volumes of $10 \%$ EDTA trisodium salt ( $\mathrm{pH} 7.5)$ under continuous stirring. The extraction mixture was centrifuged at $9,000 \times g$ for $20 \mathrm{~min}$ and the supernatant fraction containing carotenoproteins was salted out with ammonium sulfate between 20 and $50 \%$ saturation. The precipitate was dissolved in $20 \mathrm{~mm}$ potassium phosphate buffer ( $\mathrm{pH} \quad 6.8)$ containing $0.1 \mathrm{~mm}$ dithiothreitol (DTT), $0.1 \mathrm{mM}$ phenylmethyl sulfonyl fluoride (PMSF) and 0.1 mM EDTA, and dialyzed exhaustively against the same buffer.

The dialyzed solution was chromatographed on a DEAE-Toyopearl $650 \mathrm{M}$ column $(2 \times 6.5 \mathrm{~cm})$ equilibrated with $20 \mathrm{~mm}$ potassium phosphate buffer $(\mathrm{pH}$ 6.8) at a flow rate of $2 \mathrm{~m} / / \mathrm{min}$. Proteins were eluted with a linear gradient of $\mathrm{KCl}$ using $150 \mathrm{~m} l$ each of $20 \mathrm{~mm}$ potassium phosphate ( $\mathrm{pH} \mathrm{6.8)} \mathrm{and} \mathrm{the} \mathrm{same} \mathrm{buffer} \mathrm{containing} 0.3 \mathrm{M}$ 
$\mathrm{KCl}$. Elution profile was monitored by absorbance at 278 and $615 \mathrm{~nm}$. The fractions colored blue were pooled, dialyzed against the starting buffer, and rechromatographed on the same column.

The carotenoprotein fraction was further purified by high-speed gel filtration using a TSK-G 3000SWG column $(21.5 \mathrm{~mm} \times 60 \mathrm{~cm})$ equilibrated with $20 \mathrm{~mm}$ potassium phosphate $(\mathrm{pH} 6.8)$ at a flow rate of $3 \mathrm{ml} / \mathrm{min}$. The gel filtration was repeated a few times until a single peak of carotenoprotein appeared.

All steps in the purification procedure were carried out at $4^{\circ} \mathrm{C}$ in the dark or in subdued lighting.

\section{Purification of Carotenoprotein from the Muscular Epithelium}

Carotenoproteins in the muscular epithelium were extracted by treatment with collagenase. Collagenase from Clostridium histolyticum (Wako Pure Chemical, 208 units $/ \mathrm{mg}$ ) was dissolved at a concentration of $0.18 \%$ (w/ v) in $20 \mathrm{~mm}$ potassium phosphate buffer ( $\mathrm{pH} \mathrm{6.8)} \mathrm{contain-}$ ing $0.1 \mathrm{~mm}$ PMSF. To 32 abdomens without the exoskeleton $(472 \mathrm{~g}$ ) was added $720 \mathrm{ml}$ of the collagenase solution and the mixture was incubated at $28^{\circ} \mathrm{C}$ for $2 \mathrm{~h}$ under gentle shaking. After incubation, the colored solution containing carotenoproteins collected by filtration was salted out with ammonium sulfate between 20 and $60 \%$ saturation. The precipitate was dissolved in $20 \mathrm{~mm}$ potassium phosphate buffer ( $\mathrm{pH} \mathrm{6.8)}$ containing $0.1 \mathrm{~mm}$ PMSF and dialyzed exhaustively against the same buffer. Further purification was done by only high-speed gel filtration under the same conditions described above. The elution profile was monitored by absorbance at 278 and $625 \mathrm{~nm}$. All steps in the purification procedure were carried out at $4^{\circ} \mathrm{C}$ in the dark or in subdued lighting.

\section{Molecular Weight Determination}

The molecular weight was determined by high-speed gel filtration with the same conditions as for purification. The calibration curve for estimation of the molecular weight in terms of elution volume was obtained from the following standard proteins. (MW-GF-200, Sigma); $\beta$-amylase (molecular weight, $200,000=200 \mathrm{kDa}$ ), alcohol dehydrogenase $(150 \mathrm{kDa})$, bovine serum albumin $(66 \mathrm{kDa})$, carbonic anhydrase $(29 \mathrm{kDa})$, and cytochrome $c(12.4$ $\mathrm{kDa})$.

Alternatively, native polyacrylamide gel electrophoresis using a 4-20\% gradient slab gel was carried out for the carotenoprotein from the exoskeleton by the method of Davis and Ornstein. ${ }^{20)}$ Protein standards used were thyroglobulin (molecular weight, $669,000=669 \mathrm{kDa}$ ), ferritin $(443 \mathrm{kDa})$, lactate dehydrogenase $(139.9 \mathrm{kDa})$, bovine serum albumin $(66 \mathrm{kDa})$, and trypsin inhibitor from soybean $(20.1 \mathrm{kDa})$ (Daiichi Pure Chemical).

Sodium dodecyl sulfate-polyacrylamide gel electrophoresis (SDS-PAGE) using 10-20\% gradient slab gels was performed by the method of Laemmli. ${ }^{21)}$ The following standard proteins (Daiichi Pure Chemical) were used; myosin (molecular weight, $200,000=200 \mathrm{kDa}), \beta$-galactosidase $(116 \mathrm{kDa})$, bovine serum albumin $(66 \mathrm{kDa})$, aldolase $(42.4$ $\mathrm{kDa})$, carbonic anhydrase $(30 \mathrm{kDa})$ and myoglobin $(17.2$ $\mathrm{kDa})$. After running, the gel was stained with Coomassie brilliant blue R250 and scanned at $660 \mathrm{~nm}$ with a Gelman
ACD-18 densitometer to obtain the relative concentration of each subunit.

\section{Analysis of Amino Acid Composition}

The purified carotenoprotein from the exoskeleton was exhaustively dialyzed against distilled water and $0.27 \mathrm{mg}$ of the protein was hydrolyzed with $3 \mathrm{~N}$ mercaptoethanesulfonic acid at $110^{\circ} \mathrm{C}$ for $24 \mathrm{~h}$, according to the method of Penke et $a{ }^{22)}$ Amino acid composition was then analyzed using a Hitachi 835 amino acid analyzer.

\section{N-Terminal Amino Acid Sequencing}

The N-terminal amino acid sequence was determined by the method of Matsudaira ${ }^{23)}$ as follows: Proteins from the exoskeleton were separated on SDS-PAGE, electrically transferred onto an Immobilon PVDF membrane (Millipore), and stained with Coomassie brilliant blue R250. The parts of the membrane carrying the blotted bands of the carotenoprotein subunits were cut out with a clean razor. Several membranes were placed together on the Teflon seal of the cartridge block in an Applied Biosystems model $477 \mathrm{~A}$ protein sequencer with an on-line system
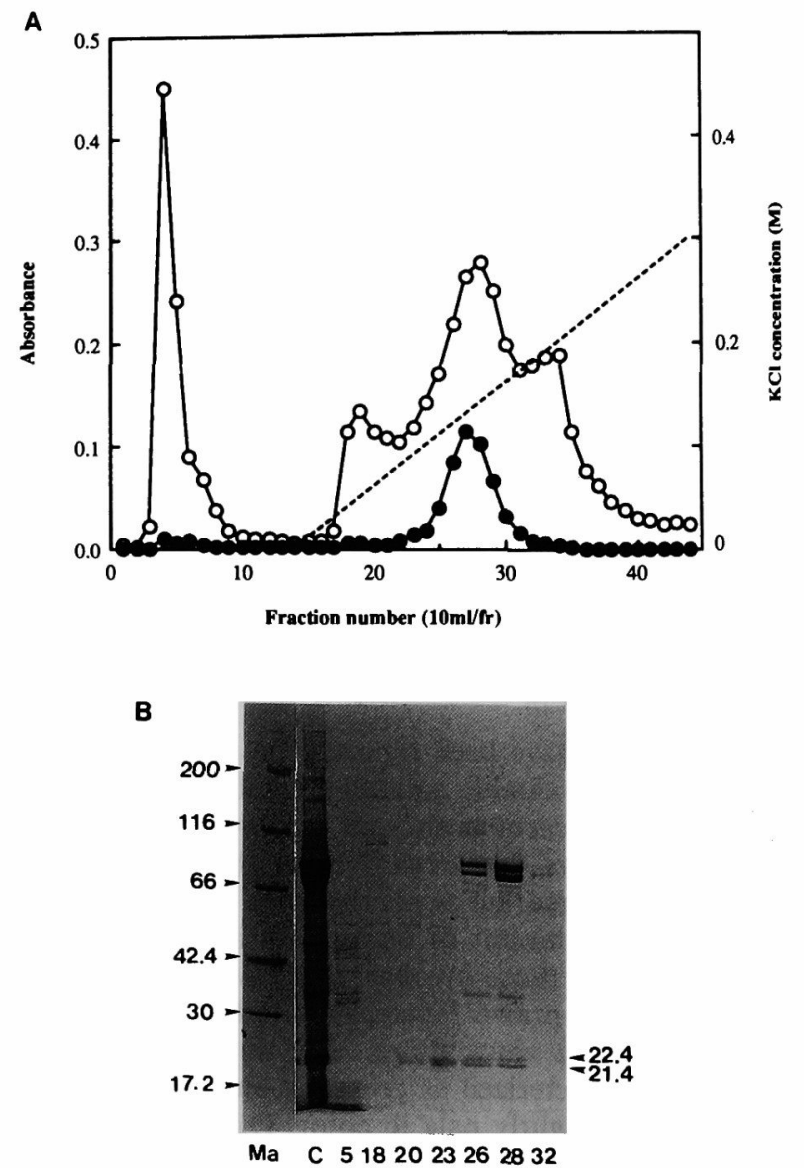

Fig. 1. A: DEAE-Toyopearl $650 \mathrm{M}$ chromatography of crude carotenoprotein from the exoskeleton of $P$. monodon.

Detection was carried out at $278 \mathrm{~nm}(O)$ and $615 \mathrm{~nm}(\bullet)$.

B: SDS-PAGE pattern of some fractions. Abbreviations used are $\mathrm{Ma}$, molecular weight markers of standard proteins; $\mathrm{C}$, crude extract. The numbers under the photograph are fraction numbers. The proteins later identified as the subunits of carotenoprotein are indicated by arrowheads ( 22.4 and $21.4 \mathrm{kDa})$. The volume of each fraction applied on a lane was $20 \mu l$. 
model $120 \mathrm{~A}$.

\section{Analysis of Carotenoid Composition}

Carotenoid was liberated by treatment with acetone from the carotenoproteins and transferred into diethyl ether according to the method of Powls and Britton. ${ }^{24)}$ The carotenoid was dissolved in benzene and the absorption spectrum was measured. Carotenoid composition was checked by thin-layer chromatography (TLC) using a silica gel $60 \mathrm{~F}_{254}$ plate (Merck) with a mobile phase of benzene/ethyl acetate (9:1). The carotenoid was also identified by high performance liquid chromatography (HPLC) on a Superiorex-ODS column $(4.6 \times 150 \mathrm{~mm})$ with a mobile phase of methanol/ethyl acetate/water (54:15:10) at a flow rate of $0.7 \mathrm{ml} / \mathrm{min}$.

\section{Determination of Stoichiometry of Carotenoid and Apoprotein}

A solution containing the purified carotenoprotein from the exoskeleton was dialyzed exhaustively against distilled water for 2 days and freeze-dried. The weight of freezedried carotenoprotein was measured gravimetrically immediately after evacuation by a vacuum pump for $1 \mathrm{~h}$. The freeze-dried carotenoprotein $(1.3 \mathrm{mg})$ was treated with ace-
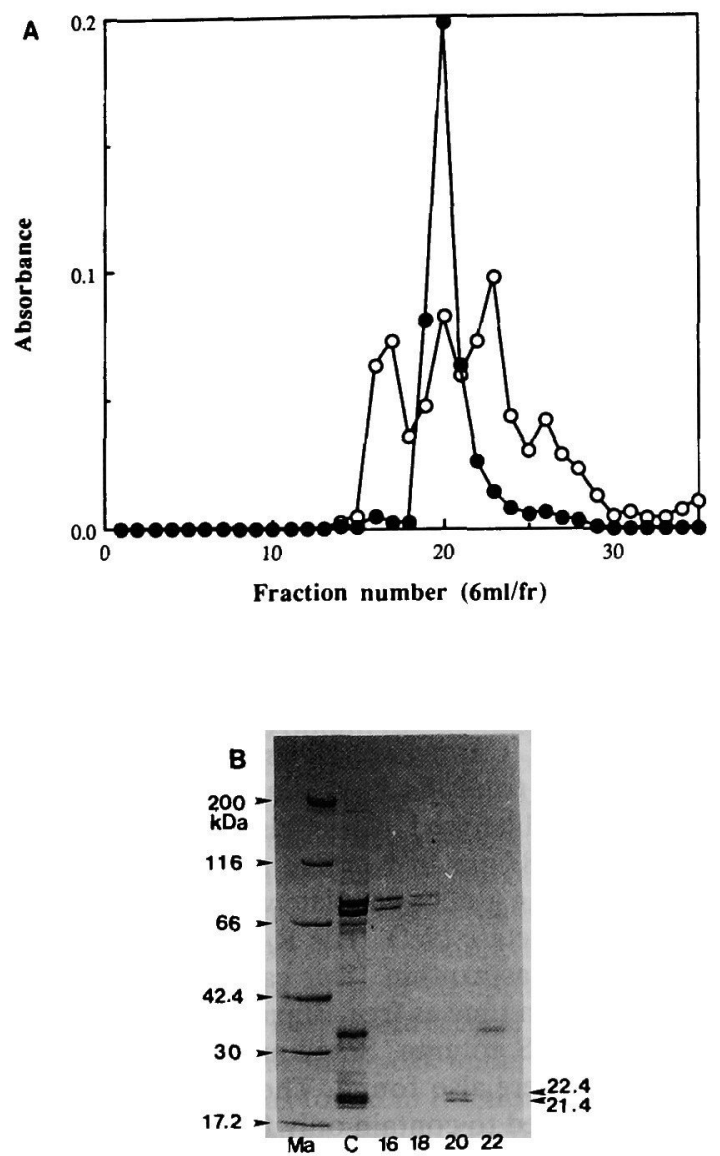

Fig. 2. A: Gel filtration on TSK G-3000SWG column of the carotenoprotein from the exoskeleton.

Detection was carried out at $278 \mathrm{~nm}(0)$ and $615 \mathrm{~nm}(\bullet)$. B: SDS-PAGE pattern of some fractions.

Refer to the legend of Fig. 1 for abbreviations and further details. The volume of each fraction applied on a lane was $20 \mu l$. tone to liberate carotenoids, and the carotenoid content was determined by the method of McBeth..$^{25}$ The molecular weight obtained by gel filtration was used for calculating the molar concentration of the carotenoprotein.

\section{Treatment of the Carotenoprotein from the Exoskeleton with Collagenase}

The purified carotenoprotein from the exoskeleton was dissolved in $20 \mathrm{mM}$ phosphate buffer ( $\mathrm{pH} 6.8$ ) and treated with collagenase in the same manner as the extraction of the carotenoprotein from the muscular epithelium. After treatment, the solution was subjected to gel filtration. Blue colored fractions were collected and any change by collagenase treatment was checked by native-PAGE.

\section{Results}

Purification of Carotenoproteins from the Exoskeleton

Figure 1A shows the elution pattern from the first DEAE-Toyopearl $650 \mathrm{M}$ column. Though roughly four peaks were observed by monitoring at $278 \mathrm{~nm}$, there was a single peak having absorbance at $615 \mathrm{~nm}$ at a concentration of $\mathrm{KCl}$ of $0.09 \mathrm{M}$. Some fractions were analyzed for protein composition pattern by SDS-PAGE. The fractions having absorbance at $615 \mathrm{~nm}$ contained mainly five components including those of 22.4 and $21.4 \mathrm{kDa}$ later identified as the subunits of the carotenoprotein (Fig. 1B). By repeating the ion exchange chromatography for the blue colored fractions, a single peak of the proteins appeared, however, the absorbance at $278 \mathrm{~nm}$ was still larger than that at 615 $\mathrm{nm}$. Then further purification was done by high-speed gel filtration. Figure $2 \mathrm{~A}$ shows the elution pattern of the proteins from the first TSK G-3000SWG column. Several peaks having absorbance at $278 \mathrm{~nm}$ appeared but there
A

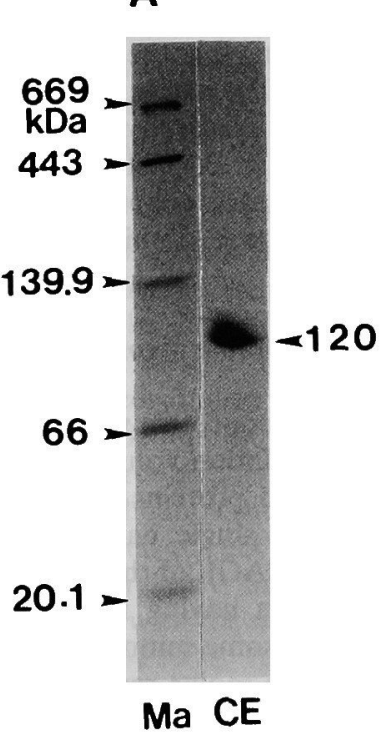

B

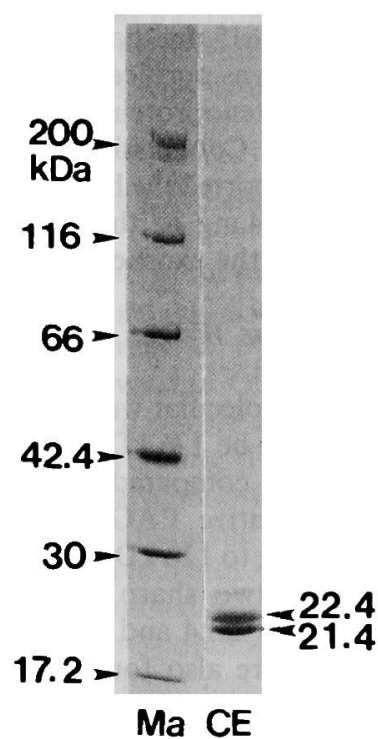

Fig. 3. A: Native-PAGE pattern of purified carotenoprotein from the exoskeleton. Ma, molecular weight markers; $\mathrm{CE}$, carotenoprotein from the exoskeleton.

B: SDS-PAGE patterm of purified carotenoprotein from the exoskeleton.

Proteins applied were $10 \mu \mathrm{g} /$ lane. 
Table 1. Amino acid composition of the carotenoprotein from the exoskeleton of $P$. monodon

\begin{tabular}{lc}
\hline \hline Amino acid & mole\% \\
\hline Arg & 4.8 \\
Lys & 5.2 \\
His & 1.4 \\
Asp & 11.5 \\
Glu & 9.1 \\
Thr & 5.2 \\
Ser & 7.7 \\
Pro & 5.6 \\
Gly & 7.8 \\
Ala & 9.3 \\
Val & 5.8 \\
Ile & 4.2 \\
Leu & 6.6 \\
Cys/2 & 1.2 \\
Met & 0.9 \\
Tyr & 6.1 \\
Phe & 6.2 \\
Trp & 0.9 \\
\hline
\end{tabular}

$\begin{array}{ll}\mathrm{PC}_{22.4} & \text { DKIPDFVVPG } \\ \mathrm{PC}_{21.4} & \text { DGIPDFVVPG } \\ \mathrm{HC}_{1} & \\ \mathrm{HA}_{2} & \text { DKIPDFVVPG }\end{array}$

Fig. 4. The N-terminal amino acid sequences of two polypeptides of $22.4 \mathrm{kDa}\left(\mathrm{PC}_{22.4}\right)$ and $21.4 \mathrm{kDa}\left(\mathrm{PC}_{21.4}\right)$ from the exoskeleton of $P$. monodon.

The sequences of apocrustacyanin $\mathrm{C}_{1}\left(\mathrm{HC}_{1}\right)^{16)}$ and apocrustacyanin $\mathrm{A}_{2}\left(\mathrm{HA}_{2}\right)^{15)}$ from $H$. gammarus are shown together.

was only one peak having absorbance at $615 \mathrm{~nm}$. SDSPAGE showed that the blue colored fractions contained two main components of 22.4 and $21.4 \mathrm{kDa}$ (Fig. 2B). After repeated gel filtration, a single peak of the blue carotenoprotein appeared and showed a higher absorbance at $615 \mathrm{~nm}$ than $278 \mathrm{~nm}$, suggesting that the carotenoprotein was purified to homogeneity. The native PAGE of this carotenoprotein showed one band irrespective of staining with Coomassie brilliant blue (Fig. 3A). This result also supported the homogeneity of the carotenoprotein. Finally, $8.4 \mathrm{mg}$ of the carotenoprotein was obtained from $110 \mathrm{~g}$ of the exoskeleton.

Molecular Weight of the Carotenoprotein from the Exoskeleton

The molecular weight of this carotenoprotein was determined to be $126 \mathrm{kDa}$ by the relative elution volume in gel filtration compared with the standard proteins. Alternatively, native PAGE showed only a single band corresponding to $120 \mathrm{kDa}$ (Fig. 3A). SDS-PAGE of this protein showed two sharp polypeptide bands having molecular weight of 22.4 and $21.4 \mathrm{kDa}$ though some minor components were also found (Fig. 3B). The molar ratio of the two subunits was about $1: 1$. These results indicated that the carotenoprotein contained six subunits, with three sets of two types of subunits of different molecular weights.

Amino Acid Composition of the Carotenoprotein from the Exoskeleton

The amino acid composition of the carotenoprotein

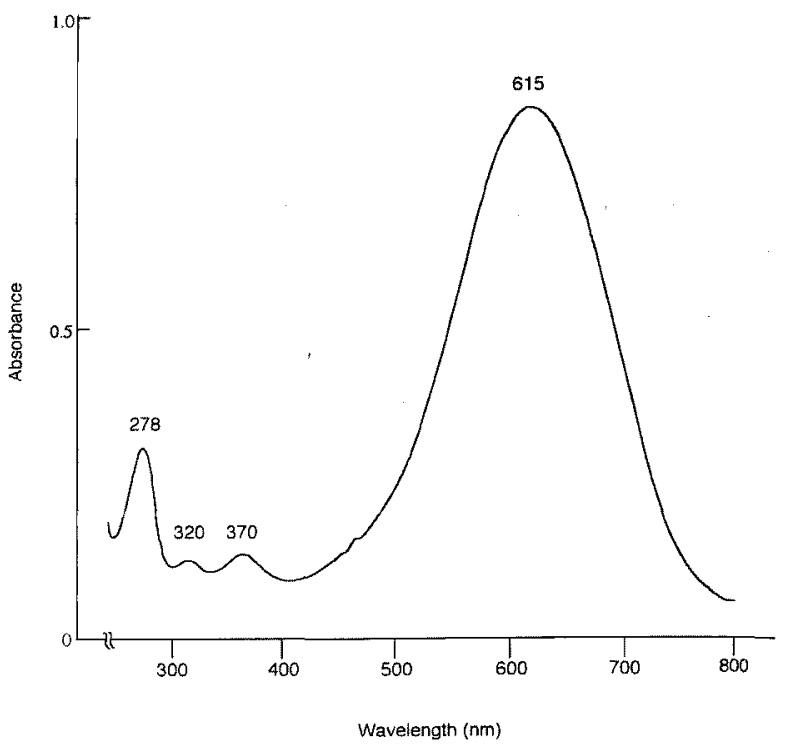

Fig. 5. Absorption spectrum of the carotenoprotein from the exoskeleton of $P$. monodon in $20 \mathrm{mM}$ phosphate buffer (pH 6.8).

from the exoskeleton is shown in Table 1. The content of aspartic acid was highest followed by alanine, glutamic acid, and glycine. The tryptophan content was about 1 mole $\%$.

\section{$N$-Terminal Amino Acid Sequence of the Carotenoprotein} from the Exoskeleton

The N-terminal amino acid sequences of the two polypeptides of 22.4 and $21.4 \mathrm{kDa}$ in the carotenoprotein from the exoskeleton are shown in Fig. 4. Ten residues were determined in both peptides. Both peptides had almost the same sequence except the second amino acid residue, which was lysine in the polypeptide of $22.4 \mathrm{kDa}$, and glycine in that of $21.4 \mathrm{kDa}$.

\section{Absorption Spectrum of the Carotenoprotein from the Ex- oskeleton}

The carotenoprotein from the exoskeleton in $20 \mathrm{mM}$ potassium phosphate buffer ( $\mathrm{pH}$ 6.8) had absorption maxima at $615,370,320$, and $278 \mathrm{~nm}$. The absorbance ratio $\left(A_{615 / 278}\right)$ was 3.67 for the purified protein (Fig. 5).

Identification of Carotenoid Prosthetic Group in the Carotenoprotein from the Exoskeleton

A carotenoid liberated from this carotenoprotein exhibited $\lambda_{\max }$ at $487 \mathrm{~nm}$ in benzene and gave a single red spot on TLC using a silica gel plate with a mobile phase of benzene/ethyl acetate $(9: 1)$. The $R_{f}$ value was coincident with that of free astaxnthin. This carotenoid also showed the same retention time as free astaxanthin in HPLC using a Superiorex ODS column. Small amounts of cis-isomers of astaxanthin were also found. Therefore, this carotenoprotein was proved to contain only free astaxanthin as the prosthetic group.

One point three milligrams of the carotenoprotein which corresponds to $1.083 \times 10^{-8}$ mole liberated $2.33 \times 10^{-8}$ mole of astaxanthin. From this result, the stoichiometry of the carotenoid and the apoprotein was determined to be a molar ratio of $2: 1$. 
A

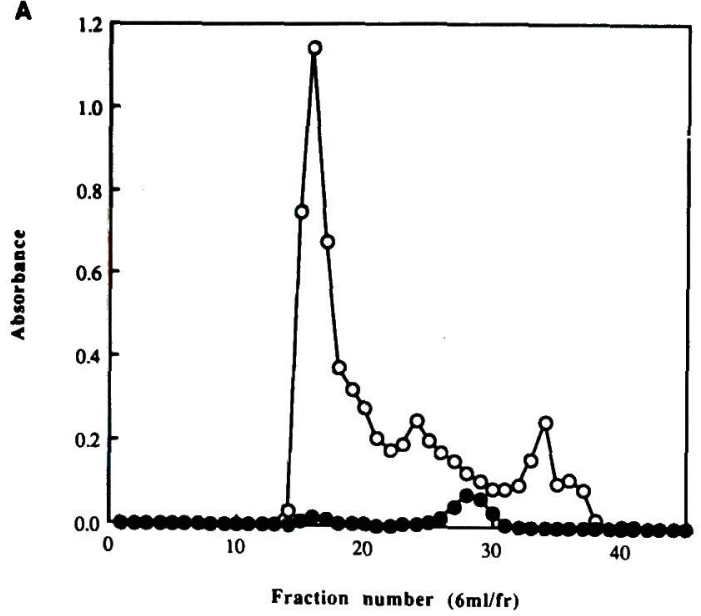

B

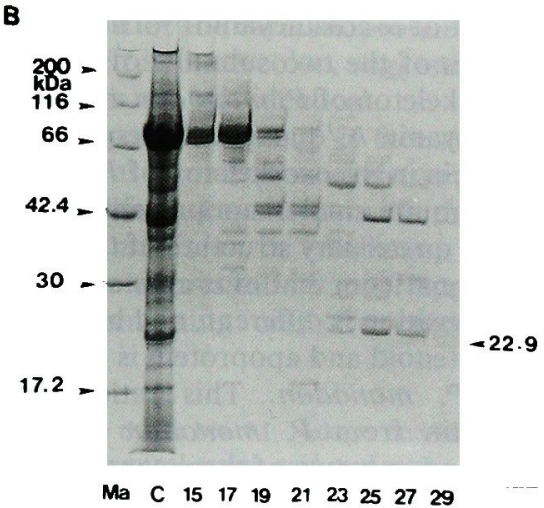

Fig. 6. A: Gel filtration on TSK G-3000SWG column of the carotenoprotein from the muscular epithelium of $P$. monodon. Detection was carried out at $278 \mathrm{~nm}(0)$ and $625 \mathrm{~nm}(\bullet)$. B: SDS-PAGE pattern of some fractions.

Refer to the legend of Fig. 1 for abbreviations and further details. The volume of each fraction applied on a lane was $20 \mathrm{\mu l}$.

\section{Purification of the Carotenoprotein from the Muscular Epithelium}

At first we failed to extract the carotenoprotein in the muscular epithelium by the same method as the extraction of carotenoprotein from the exoskeleton. Collagenase treatment enabled us to obtain the soluble carotenoprotein from the muscular epithelium with its color intact. This carotenoprotein was not retained on the DEAE Toyopearl column equilibrated with $20 \mathrm{~mm}$ phosphate buffer. Therefore, further purification was carried out by only highspeed gel filtration. Figure 6A shows the elution pattern of the first gel filtration of the carotenoprotein. Some fractions were analyzed for protein composition pattern by SDS-PAGE (Fig. 6B). There were several peaks having absorbance at $278 \mathrm{~nm}$. The main peak under absorption measurements at $625 \mathrm{~nm}$ was found in fraction No. 25 to 31 . A minor peak was also found around fraction No. 15 to 18 . This minor peak eluting around the void volume may be caused by carotenoids liberated and bound artificially to aggregated proteins during precipitation with ammonium sulfate or subsequent dialysis, because the color of those fractions was not blue for carotenoproteins but red for free carotenoids. The retarded fractions were collected as
A

B
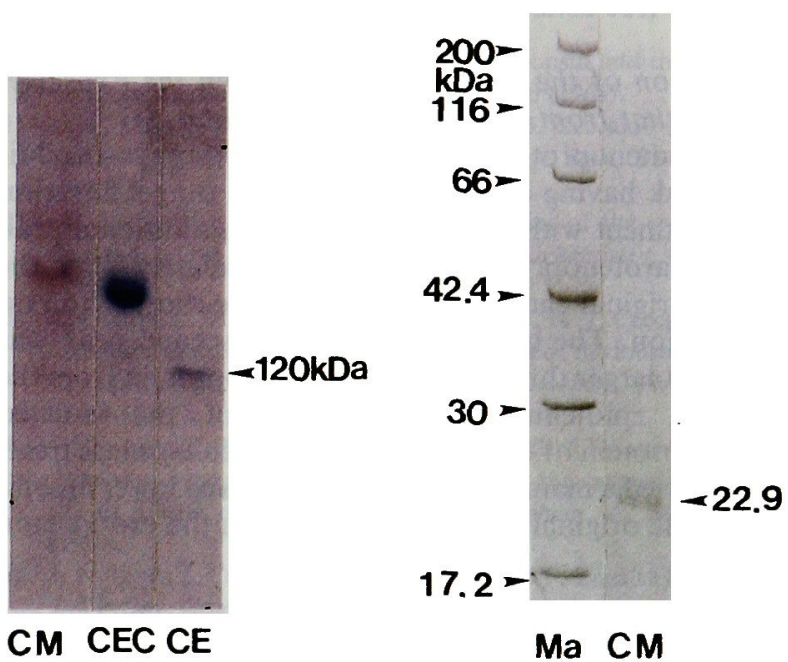

Fig. 7. A: Native-PAGE pattern of purified carotenoprotein from the muscular epithelium, carotenoprotein from the exoskeleton, and carotenoprotein from the exoskeleton after collagenase treatment.

The gel is not stained with Coomassie brilliant blue. Abbreviations used are CM, carotenoprotein from the muscular epithelium; CEC, a newly derived carotenoprotein from the original carotenoprotein from the exoskeleton after collagenase treatment; $\mathrm{CE}$, carotenoprotein from the exoskeleton. Proteins applied were 5 $\mu \mathrm{g} /$ lane (CM), $10 \mu \mathrm{g} /$ lane (CEC), $2 \mu \mathrm{g} /$ lane (CE).

$\mathrm{B}$ : SDS-PAGE pattern of purified carotenoprotein from the muscular epithelium.

Proteins applied were $2 \mu \mathrm{g} /$ lane.

carotenoproteins, though the absorbance at $625 \mathrm{~nm}$ was smaller than that at $278 \mathrm{~nm}$, and subjected to the same gel filtration repeatedly. The carotenoprotein was isolated as a single peak having absorbance at $625 \mathrm{~nm}$ after three repetitions of the gel filtration. The yield was $3.3 \mathrm{mg}$ from $32 \mathrm{ab}$ dominal parts $(472 \mathrm{~g})$.

\section{Molecular Weight Determination of the Carotenoprotein} from the Muscular Epithelium

The molecular weight of this carotenoprotein was determined to be $21.4 \mathrm{kDa}$ by high-speed gel filtration. The molecular weight could not be determined by native PAGE according to the method of Davis and Ornstein ${ }^{20}$ because this blue carotenoprotein turned purple and gave at least three pigmented bands during running (Fig. 7A). Additional bands without the color of carotenoprotein appeared when the gel was stained with Coomassie brilliant blue. The relative mobilities of the purple bands were not consistent with the molecular size determined by gel filtration. SDS-PAGE showed a band corresponding to the molecular weight of $22.9 \mathrm{kDa}$ but the band was obscure (Fig. 7B).

\section{Identification of Carotenoid Prosthetic Group of the Carotenoprotein from the Muscular Epithelium}

The carotenoid liberated from this carotenoprotein gave a single red spot on TLC using a silica gel plate with a mobile phase of benzene/ ethyl acetate (9:1) and the $R_{f}$ value 
was coincident with that of free astaxanthin. The carotenoid had a maximum absorbance at $487 \mathrm{~nm}$ in benzene. From these results, this carotenoprotein was proved to have only free astaxanthin as the prosthetic group.

\section{Comparison of the Carotenoproteins from the Exoskele- ton with that from the Muscular Epithelium}

The carotenoprotein from the exoskeleton gave an additional peak having absorbance at $625 \mathrm{~nm}$ on gel filtration after treatment with the collagenase. The additional peak of blue carotenoprotein appeared immediately after the peak of original carotenoprotein from the exoskeleton on gel filtration. The molecular size of the second peak was somewhat larger than that of the carotenoprotein from the muscular epithelium. Native-PAGE of the isolated carotenoprotein of the second peak after collagenase treatment showed a main blue band which showed lower mobility than the original carotenoprotein from the exoskeleton (Fig. 7A).

\section{Discussion}

The absorption maximum at $615 \mathrm{~nm}$ of the carotenoprotein from the exoskeleton was somewhat red-shifted compared with the corresponding value for the blue carotenoproteins from $P$. japonicus ${ }^{18)}$ and $\alpha$-crustacyanin from lobster. ${ }^{12)}$ It shifted from $615 \mathrm{~nm}$ to around $480 \mathrm{~nm}$ by heating the carotenoprotein to $50^{\circ} \mathrm{C}$ (data not shown). This large shift suggests a strong carotenoid-protein interaction which is frequently found in blue carotenoproteins isolated from other crustaceans. The purification procedure adopted in this study for the carotenoprotein from the exoskeleton was almost the same as that for $\alpha$-crustacyanin from the lobster Homarus gammarus. ${ }^{26)}$ The carotenoprotein from the exoskeleton of $P$. monodon was eluted from a DEAE column at a lower concentration of $\mathrm{KCl}$ than $\alpha$ or $\gamma$-crustacyanin. This means that the carotenoprotein from $P$. monodon is less negativelycharged than crustacyanins from $H$. gammarus. The absorption ratio $\left(\mathrm{A}_{615 / 278}\right)$ of 3.67 is quite similar to that of crustacyanin purified from $H$. gammarus. ${ }^{12)}$ This value is consistent with the amino acid composition which shows similar abundance of tyrosine and phenylalanine to crustacyanin. ${ }^{13)}$ Some of these aromatic amino acids are thought to be important in stabilizing the binding of astaxanthin in the cavity of apocrustacyanin. ${ }^{16)}$ The carotenoprotein from the exoskeleton of $P$. monodon may have the same mechanism to stabilize the binding between carotenoid and apoprotein by these aromatic amino acids.

On the other hand, the carotenoprotein in the muscular epithelium of $P$. monodon was not extracted in the same way for the exoskeleton. Muriana et al. extracted a carotenoprotein of $P$. japonicus from the hypodermis with phosphate buffer, though the carotenoprotein obtained showed a quite large absorption maximum at $276 \mathrm{~nm} .{ }^{18)}$ It is not known whether the difficulty in extraction of the carotenoprotein in the muscular epithelium of $P$. monodon is owing to its original properties or denaturation by freezing. The carotenoprotein from the muscular epithelium was not adsorbed onto a DEAE column at all. The collagenase treatment might deprive the carotenoprotein of charging effects. The absorption spectrum of the carotenoprotein from the muscular epithelium has an absorption maximum at $625 \mathrm{~nm}$ quite similar to those of other blue carotenoproteins. ${ }^{4,8,9)}$

The molecular weight of the carotenoprotein from the exoskeleton of $P$. monodon is rather smaller than that of $\alpha$-crustacyanin $(380 \mathrm{kDa})^{12)}$ or a blue carotenoprotein from $P$. japonicus $(280 \mathrm{kDa}){ }^{18)}$ In native PAGE, the carotenoprotein from $P$. monodon was so homogenous that it showed only one band $(120 \mathrm{kDa})$. The results from SDSPAGE indicated that this carotenoprotein contained six subunits, with three sets of two subunits of molecular weight $22.4 \mathrm{kDa}$ and $21.4 \mathrm{kDa}$. A minor band of $23.1 \mathrm{kDa}$ was also seen, but the appearance of this band depended on the preparation. This component might be a byproduct derived from one of the two major components during purification. The sizes of two subunits are very close to those of apocrustacyanins $A_{1}, C_{1}, C_{2}(23 \mathrm{kDa})$ and $\mathrm{A}_{2}, \mathrm{~A}_{3}(21.5 \mathrm{kDa}) .{ }^{26)}$ The amino acid composition also resembles that of $\alpha$-crustacyanin. ${ }^{13)}$ The $\mathrm{N}$-terminal amino acid sequences of the two subunits of 21.4 and $22.4 \mathrm{kDa}$ from the exoskeleton of $P$. monodon are also quite similar to apocrustacyanin $\mathrm{A}_{2}{ }^{15)}$ and $\mathrm{C}_{1}{ }^{16)}$ respectively. Thus, the carotenoprotein in the exoskeleton of $P$. monodon may be composed of quite similar apoproteins as crustacyanins. However, the quaternary structure of this carotenoprotein must be different from that of $\alpha$-crustacyanin, because the subunit composition is different, and because the stoichiometry of carotenoid and apoprotein is $2: 1$ in the carotenoprotein of $P$. monodon. This ratio means that the carotenoprotein from $P$. monodon is composed of 6 apoproteins and only two of them contain one molecule of astaxanthin. On the other hand, $\alpha$-crustacyanin is an octamer of $\beta$-crustacyanin which is composed of one set of two types of apocrustacyanin having one molecule of astaxanthin. ${ }^{6)}$ The total number of astaxanthin molecules in the carotenoprotein from the exoskeleton of $P$. monodon is the same as that of $\beta$-crustacyanin though the size of this carotenoprotein is three times as large as $\beta$ crustacyanin. Tryptophan residue is thought to be important in stabilizing astaxanthin molecules in a cavity of apocrustacyanin. ${ }^{13)}$ One tryptophan residue is thought to be necessary to stabilize one molecule of astaxanthin. The number of tryptophan residue in the carotenoprotein from the exoskeleton of $P$. monodon is rather smaller than that in $\alpha$-crustacyanin. ${ }^{13,15,16)}$ This may explain the smaller number of astaxanthin molecules in the carotenoprotein from the exoskeleton of $P$. monodon than $\alpha$-crustacyanin.

The molecular weight of the carotenoprotein from the muscular epithelium is rather smaller than that from the exoskeleton or that from the hypodermis of $P$. japonicus. ${ }^{187}$ The molecular weight determined by gel filtration is very close to that of a subunit determined by SDS-PAGE, indicating that this carotenoprotein is composed of only one polypeptide chain. It is not clear why this epitherial blue carotenoprotein turned purple and gave many bands in native-PAGE. This phenomenon seems to resemble the dissociation of $\alpha$-crustacyanin to $\beta$-crustacyanin which is purple and electrophoretically heterogeneous. But the molecular size of the carotenoprotein from the muscular epithelium of $P$. monodon is about $20 \mathrm{kDa}$ and corresponds to one apocrustacyanin. Therefore, it is unlikely that this carotenoprotein dissociates into smaller compo- 
nents. The many bands on native-PAGE of the carotenoprotein from the muscular epithelium may mean the presence of same size proteins with different charge effects formed by collagenase treatment because SDS-PAGE of this carotenoprotein showed a single band of $22.9 \mathrm{kDa}$.

Another explanation for the curious pattern of the bands in native-PAGE is variation in the molecular weight of the carotenoprotein by aggregation. Some parts of the original carotenoprotein might be deprived by collagenase treatment, resulting in changes of intact configuration. Such a structural change could have led to aggregation of the carotenoprotein by exposing its hydrophobic parts.

The carotenoprotein from the exoskeleton also underwent some change by collagenase treatment. Its molecular weight determined by gel filtration is not consistent with that determined by the method of Davis and Ornstein. ${ }^{20}$ ) The effect of collagenase on both carotenoproteins from the exoskeleton and the muscular epithelium should be same. However, most of the carotenoprotein from the exoskeleton maintained its blue color, suggesting that these two carotenoproteins are not identical.

The carotenoid prosthetic group is free astaxanthin in both carotenoproteins from the exoskeleton and the muscular epithelium. This is consistent with the carotenoid composition of $P$. monodon. ${ }^{19,27)}$ Different colors of $P$. monodon might be expressed by overlapping of blue carotenoproteins containing only free astaxanthin and astaxanthin esters.

Acknowledgements We thank Ms. M. Nakaya and Mr. Y. Hirayama for helping with the N-terminal amino acid sequencing. This study was partly funded by a Grant-in-Aid from the Ministry of Education, Science, and Culture of Japan.

\section{References}

1) D. F. Cheesman, W. L. Lee, and P. F. Zagalsky: Carotenoproteins in invertebrates. Biol. Rev., 42, 131-160 (1967).

2) P. F. Zagalsky: Carotenoid-protein complexes. Pure Appl. Chem., 47, 103-120 (1976)

3) P. F. Zagalsky, H. J. Ceccaldi, and R. Daumas: Comparative studies on some decapod crustacean carotenoproteins. Comp. Biochem. Physiol., 34, 579-607 (1970).

4) L. Wahlgren-Brannstom and M. Baltscheffsky: Effects of ionic strength and $\mathrm{pH}$ on the visible absorption spectrum of crustacyanin from Homarus vulgaris. Acta Chem. Scand., B33, 613-614 (1979).

5) W. P. Jencks and B. Buten: The denaturation of crustacyanin. Arch. Biochem. Biophys., 107, 511-520 (1964).

6) P. F. Zagalsky, E. E. Eliopoulos, and J. B. C. Findlay: The architecture of invertebrate carotenoproteins. Comp. Biochem. Physiol., 97B, 1-18 (1990).

7) P. F. Zagalsky and R. Jones: Quaternary structures of the astaxanthin-proteins of Velella velella, and of $\alpha$-crustacyanin of lobster carapace, as revealed in electron microscopy. Comp. Biochem. Physiol., 71B, 237-242 (1982).

8) A. M. Garate, E. Urrechaga, J. C. G. Milicua, R. Gomez, and G. Britton: A blue carotenoprotein from the carapace of the crab, $\mathrm{Car}$ - cinus maenas. Comp. Biochem. Physiol., 77B, 605-608 (1984).

9) A. Villarroel, A. M. Garate, R. Gomez, and J. C. G. Millicua: A blue carotenoprotein from Upogebia pusill. Purification, characterization and properties. Comp. Biochem. Physiol., 81B, 547-550 (1985).

10) T. Yoshizawa and G. Wald: Pre-lumirhodopsin and the bleaching of visual pigments. Nature, 197, 1279-1286 (1963).

11) D. F. Cheesman, P. F. Zagalsky, and H. J. Ceccaldi: Purification and properties of crustacyanin. Proc. Roy. Soc., B164, 130-151 (1963).

12) M. Buchwald and W. P. Jencks: Properties of the crustacyanins and the yellow lobster shell pigment. Biochemistry, 7, 844-859 (1968).

13) R. Quarmby, D. A. Norden, P. F. Zagalsky, H. J. Ceccaldi, and R. Daumas: Studies on the quaternary structure of the lobster exoskeleton carotenoprotein, crustacyain. Comp. Biochem. Physiol., 56B, 55-61 (1977).

14) J. B. Clarke, E. E. Eliopoulos, J. B. C. Findlay, and P. F. Zagalsky: Alternative ligands as probes for the carotenoid-binding site of lobster carapace crustacyanin. Biochem. J., 265, 919-921 (1990).

15) J. N. Keen, I. Caceres, E. E. Eliopoulos, P. F. Zagalsky, and J. B. C. Findlay: Complete sequence and model for the $A_{2}$ subunit of the carotenoid pigment complex, crustacyanin. Eur. J. Biochem., 197, 407-417 (1991).

16) J. N. Keen, I. Caceres, E. E. Eliopoulos, P. F. Zagalsky, and J. B. C. Findlay: Complete sequence and model for the $C_{1}$ subunit of the carotenoprotein, crustacyanin, and model for the dimer, $\beta$-crustacyanin, formed from the $C_{1}$ and $A_{2}$ subunits with astaxanthin. Eur. J. Biochem., 202, 31-40 (1991).

17) P. F. Zagalsky, E. E. Eliopoulos, and J. B. C. Findlay: The lobster carapace carotenoprotein, $\alpha$-crustacyanin. Biochem. J., 274, 79-83 (1991).

18) F. J. G. Muriana, V. Ruiz-Gutierrez, M. L. Gallardo-Guerrero, and M. I. Minguez-Mosquera: A study of the lipid and carotenoprotein in the prawn, Penaeus japonicus. J. Biochem., 114, 223229 (1993)

19) S. Okada, S. A. Nur-E-Borhan, and K. Yamaguchi: Carotenoid composition in the exoskeleton of commercial black tiger prawns. Fisheries Sci., 60, 213-215 (1994).

20) B. J. Davis and L. Ornstein: Disc gel electrophoresis. Ann. N. Y. Acad. Sci., 121, 404-427 (1964).

21) U. K. Laemmli: Cleavage of structural proteins during the assembly of the head of bacteriophage $T_{4}$. Nature, 227, 680-685 (1970).

22) B. Penke, R. Ferenczi, and K. Kovacs: A new acid hydrolysis method for determinating tryptophan in peptides and proteins. Anal. Biochem., 60, 45-50 (1974).

23) P. Matsudaira: Sequence from picomole quantities of protein electroblotted onto polyvinylidene difluoride membranes. J. Biol. Chem., 262, 10035-10038 (1987).

24) R. Powls and G. Britton: A carotenoprotein containing violaxanthin isolated from Scenedesmus obliquus $\mathrm{D}_{3}$. Biochim. Biophys. Acta, 453, 270-276 (1976).

25) J. W. McBeth: Carotenoid from nudibranchs. Comp. Biochem. Physiol., 41B, 55-68 (1972).

26) P. F. Zagalsky: Invertebrate carotenoproteins, in "Method in Enzymology" (ed. by J. H. Caw and H. C. Rilling), Vol. 111, Academic Press, San Diego, 1985, pp. 216-247.

27) B. K. Howell and A. D. Matthews: The carotenoids of wild and blue disease affected farmed tiger shrimp (Penaeus monodon, Fabricus). Comp. Biochem. Physiol., 98B, 375-379 (1991). 